\title{
Relación de algunos correlatos biológicos y demográficos con la práctica físico-deportiva en estudiantes universitarios. El caso de la Universidad de Guadalajara, México.
}

\section{Relationship between some biological and demographics factors with physical-sport practice in college students. A case study of Guadalajara University, Mexico.}

\author{
Gabriel Flores Allende \\ Facultad de Cultura Física y Deportes, Universidad de Guadalajara, México \\ Francisco Ruiz Juan \\ María Elena García Montes \\ Facultad de Ciencias del Deporte. Universidad de Murcia, España
}

\section{Resumen}

La práctica físico-deportiva de tiempo libre de los jóvenes universitarios, no se ajusta suficientemente a las recomendaciones de un estilo de vida activo que sea favorable para su salud. Los estudios coinciden en que algunos correlatos biológicos y demográficos, se asocian con la adherencia y continuidad de participación de los jóvenes, en el ejercicio físico y el deporte. Sugieren su identificación para desarrollar y facilitar eficaces intervenciones, con adecuados programas que fomenten la participación de los sujetos. Los estudiantes de Educación Superior de la Universidad de Guadalajara, México ( $n=1.207)$, fueron seleccionados para llevar a cabo el estudio. Haciendo uso del cuestionario, se valoró qué sectores de la población realizan práctica, los que habiendo sido activos han dejado de serlo, y los que nunca han participado en su tiempo libre. Entre los principales resultados, destacar que poco más de la mitad de los jóvenes son practicantes, siendo más probables los hombres respecto a las mujeres, siendo éstas más factibles al abandono y a no haber participado nunca en la práctica. Descartando asociaciones entre la actividad físico-deportiva con la edad, el sobrepeso-obesidad (IMC) y lesiones o días de enfermedad, en esta población. Resultando provechoso para evitar el fracaso en el diseño de programas y promover, los beneficios saludables que permite la actividad física y el deporte.

Palabras clave: factores biológicos y demográficos; práctica físico-deportiva; estudiantes universitarios.

\section{Abstract}

Leisure-time physical - sports practice of college students, does not adjust sufficiently to the recommendations of an active lifestyle that enhance their health. The studies coincide that some biological and demographic factors, associated in the adherence and continuity of participation of college students, in the physical exercise and the sport. They suggest their identification to develop and to facilitate effective interventions, with suitable programs that foment the participation of the subjects. College students of the University of Guadalajara, Mexico ( $n=1.207)$, were selected to carry out the study. Using the questionnaire, there were valued the sectors of the population that realize practice, which having been active it have stopped being, and those who have never joined leisure time physical activity. Between the principal results, to emphasize that little more than the half of college students join leisure time physical activity, being more probable the men with regard to the women, being these more feasible to the retire and to never have taken part in the practice. Rejecting associations between the physical - sports activity with the age, the overweight-obesity (BMI) and injuries or days of disease, in this population. Turning out to be profitable to avoid the failure in the design of programs and to promote, the healthy benefits that the physical activity and the sport allows.

Key words: biological and demographic factors; university students; physical activity.

Correspondencia/correspondence: Gabriel Flores Allende

Facultad de Ciencias del Deporte, Universidad de Murcia (Campus de San Javier)

Argentina s/n - Santiago de la Ribera, San Javier - Murcia

E-mail: cfdgesa75@hotmail.com 
Flores, G.; Ruiz, F.; García, M.E. (2009). Relación de algunos correlatos biológicos y demográficos con la práctica físico-deportiva en estudiantes universitarios. El caso de la Universidad de Guadalajara, México.

\section{Introducción}

E n los últimos años, los estudios sobre la actividad física en la vida cotidiana y ocio, han cobrado importancia, por los grandes beneficios que ésta origina, concediendo a los sujetos ser más saludables y mejorar su calidad de vida.

Los estudios epidemiológicos (en su mayoría sobre población adulta) muestran que los sujetos que realizan actividades físicas de forma regular, favorecen positivamente su salud física (Jacoby, Bull y Neiman, 2003; U.S Department of Health and Human Services, 1996; Varo Cenarruzabeitia, Martínez-González y Martínez Hernández, 2003), mental (Biddle, 1993; Brown y Siegel, 1988; Dechavanne, 1998; Guillén García, Castro Sánchez y Guillén García, 1997; Pérez, 1998) y social (Benaziza, 1998).

Para lograr beneficios saludables, los sujetos deben acumular por lo menos treinta minutos de actividad física moderada o veinte minutos de actividad física vigorosa preferiblemente todos los días de la semana, en ambos casos (Pate, Pratt, Blair, Hakell, Macera, Bouchard et al., 1995; USDHHS, 1996 y 2000). Con una intensidad del 65-90\% del ritmo cardíaco máximo o $50 \%-80 \%$ de la reserva de la frecuencia cardíaca, será suficiente para mejorar la aptitud cardiorrespiratoria (Pollock, Gaesser, Butcher, Després, Dishman, Franklin et al., 1998). Esto supondrá la reducción en la aparición de enfermedades, como por ejemplo en el caso de las cardíacas coronarias que afectan a los hombres de mediana edad, más proclives a sufrir estos males (Léon, Connett, Jacobs y Rauramaa, 1987).

En otras palabras, realizar y mantener niveles razonables de actividad físico-deportiva disminuye de forma considerable la aparición de algunos padecimientos crónicos o muerte prematura (Márquez, Rodríguez y Dechavanne, 2006; Organización Mundial de la Salud, 2002; USDHHS, 1996), incluso cuando son realizadas en la vida cotidiana (Granero Gallegos, Ruiz Juan y García Montes, 2005).

Sin embargo a pesar de las claras ventajas, la carencia de actividad física en el estilo de vida de la población universitaria, ha sido un problema de salud pública (Kilpatrick, Hebert y Bartholomew, 2005), debido a la disminución en la participación. Siendo necesario conocer qué factores inducen, facilitan o refuerzan la adherencia a la práctica, con el propósito de que los jóvenes adquieran un estilo de vida activo por largos periodos de tiempo.

Según varias investigaciones (Dishman y Sallis, 1994; Dishman, Sallis y Orenstein 1985; Sallis y Owen, 1999; Trost, Owen, Barman, Sallis y Brown, 2002), la práctica de actividad física en el tiempo libre puede ser afectada o favorecida por correlación de algunas variables que pueden condicionar la adherencia, continuidad y participación de las personas. Dichos estudios coinciden en señalar que los factores biológicos y demográficos; psicológicos, cognitivos y emocionales; atributos conductuales y habilidades; características de la actividad física, sociales y culturales, físico ambiental, son algunos determinantes o correlatos que han sido identificados como aspectos que producen cambios en el estado del ejercicio, presentando influencias significativas en el comportamiento de la salud. Estos determinantes también pueden ser considerados punto de partida en la promoción de estilos de vida saludables (Chillón, Tercedor, Delgado y González-Gross, 2002).

Para Sallis (1999) los factores biológicos y demográficos, guardan una asociación muy elevada respecto al nivel de actividad física, ocasionado por diferentes causas que hacen variar la actividad; destacan el sexo, la edad, las lesiones, sobrepeso/obesidad, Índice de Masa Corporal (IMC), el estado civil, los ingresos económicos y la ocupación. Sin embargo, no todos fac- 
Flores, G.; Ruiz, F.; García, M.E. (2009). Relación de algunos correlatos biológicos y demográficos con la práctica físico-deportiva en estudiantes universitarios. El caso de la Universidad de Guadalajara, México.

tores actúan en similitud de circunstancias o manera uniforme, debido a la variabilidad de las poblaciones y periodos de tiempo (Dishman et al., 1985). Por ejemplo, las personas con estudios superiores, tienen una mayor probabilidad de realizar práctica, respecto a aquellas que poseen bajo nivel de estudios (Barnes y Schoenborn, 2003), sucediendo de igual manera en la situación laboral (profesional-obrera) y estado socioeconómico (Parks, Houseman, y Browson, 2003), resultando inadecuado indagar sobre estos correlatos con la práctica entre los jóvenes universitarios. Dado que algunos correlatos tiene mayor relación o relevancia en poblaciones en particular y menor en los jóvenes.

Tomando estas consideraciones resulta provechoso analizar algunos de los correlatos que integran los factores biológicos y demográficos, particularmente el sexo, la edad, las lesiones y el sobrepeso u obesidad, para observar si estos conducen a la actividad o inactividad de práctica físico-deportiva de tiempo libre en nuestros jóvenes universitarios.

\section{Sexo}

El sexo es un correlato que tiene una potente asociación con la participación en actividades físicas (Sallis, 1999), pues son las mujeres quienes habitualmente se muestran menos activas que los hombres, ocurriendo de igual manera en estudios sobre poblaciones universitarias (Hernández Rodríguez, 2001; Kelly y Sharpe, 1994; Leslie, Owen, Salmon, Bauman, Sallis y Lo, 1999; Ruiz Juan, García Montes y Hernández, 2001; Ruiz Juan, García Montes y Gómez López 2005; Sánchez Pérez, García Domínguez, Landabaso y Nicolás Martínez, 1998). Aunque otros muestran lo contrario (Von Bothmer y Fridlun, 2005), al no encontrar diferencias significativas entre sexos.

Aunque los resultados muestran que los hombres se implican más que las mujeres en el ejercicio físico y el deporte, las diferencias porcentuales varían. Es decir, no siempre son las mismas, oscilando desde un quince a un treinta por ciento. Es claramente remarcable, las propias discrepancias entre los porcentajes de hombres practicantes, que alcanzan hasta cuarenta y cinco unidades porcentuales, y de mujeres que realizan actividad física, que asciende hasta treinta y dos; lo que implica que las cifras de mujeres practicantes, iguala e incluso supera, en algunos casos, las de los hombres.

En relación a este último planteamiento, los resultados obtenidos por Ruiz Juan et al. (2005), muestran evoluciones en los índices de práctica del alumnado universitario de primer ciclo, transcurridos cinco años (1996-1997), en contraste con los datos de Hernández Rodríguez (2001), y de segundo ciclo, tres años después (1998-1999), en relación a las aportaciones de Ruiz Juan et al. (2001). En dicha investigación se observan modificaciones con el paso del tiempo, en los porcentajes de práctica de hombres y mujeres, que acortan las diferencias existentes entre unos y otras. Así, mientras en primer ciclo disminuye la práctica de los chicos en un $10 \%$ manteniéndose igual la de las chicas, en segundo ciclo el descenso entre ellos duplica el que se produce entre ellas, mermando, en ambos casos, las disimilitudes de sujetos activos de ambos sexos, aunque no llegan a equilibrarse. Esto despierta la preocupación sobre los abandonos de la actividad física, como forma de ocupar el tiempo libre, en esta etapa, pero parece hacer vislumbrar un afianzamiento de las mujeres de este colectivo universitario. 
Flores, G.; Ruiz, F.; García, M.E. (2009). Relación de algunos correlatos biológicos y demográficos con la práctica físico-deportiva en estudiantes universitarios. El caso de la Universidad de Guadalajara, México.

Revista Internacional de Ciencias del Deporte. 14(5), 59-80. http://www.cafyd.com/REVISTA/01406.pdf

\section{Edad}

Otro correlato bastante abordado ha sido la edad, considerado un potente predictor relacionado con la práctica, pues conforme ésta aumenta, tienden a presentarse disminuciones, sucediendo principalmente en la etapa de adolescencia y juventud. Algunos Expertos señalan que a partir de los doce años comienza el declive (Telama y Yang, 2000), en tanto para otros ocurre entre los trece y dieciocho años (Sallis, 2000). Para este último la disminución resulta más probable en hombres en la juventud y las mujeres en la adolescencia, desde luego dependiendo del tipo y la intensidad de la actividad física.

Pocas investigaciones en poblaciones universitarias abordan esta variable, debido a la diversidad en grupos de edad que se encuentran en estas etapas. Al respecto Keating, Guan, Castro y Bridges (2005), enfatizan que la mayoría de los estudios en periodos previos a la universidad tienen superiores condiciones en la agrupación de los sujetos por la similitud en los grupos de edad, permitiendo observar tendencias. En cambio en estos sectores se presenta diversos grupos de edad, dificultando así la integración de grupos o categorías comunes que imposibilitan realizar comparaciones a través de la literatura existente. Aunque destaca que la edad media es de veinte años.

Entre los pocos trabajos que tratan esta variable destaca el realizado por Leslie, Sparling y Owen (2001), sobre las diferencias relativas a la edad (18 a 19, 20 a 24 y 25 a 29) en comportamientos de los jóvenes universitarios en la práctica de actividad física de tiempo libre. Este grupo de investigadores resalta que los jóvenes de menor edad fueron los sujetos que presentaban superioridad con respecto a los otros grupos en las actividades físicas vigorosas, moderadas e insuficientes. En tanto, resultados como los obtenidos por Ruiz Juan et al. (2001), resaltan que el grupo de universitarios (mayores de 21 años) que dos cursos académicos atrás, confirmaba la tendencia que afirma que conforme aumenta la edad ocurren disminuciones de la práctica; dos periodos después, la situación fue totalmente contraria.

En esta misma línea, tiempo después Ruiz Juan et al. (2005), analiza la evolución de los comportamientos de la práctica de actividad física en el curso académico 2000-2001 del alumnado de primer y segundo ciclo de la Universidad de Almería, transcurridos cinco (1996-1997) y tres (1998-1999) años respectivamente. Al tratar la variable edad observa que los alumnos más jóvenes de 18 a 20 años de primer ciclo habían disminuido en la práctica con el paso de los años, en tanto, el grupo mayor de veintiún años mantenía los mismos valores porcentuales de tiempo atrás. En cambio, con los alumnos de segundo ciclo sucedía lo contrario, pues el grupo más joven, mantenía los mismos valores porcentuales de tiempo atrás, pero el colectivo mayor de veintiún años con el paso de los años, había caído hasta en un 50\%. Mostrando la dificultad de confirmar tendencias de disminución de la práctica, respecto al incremento de la edad en este sector de la población.

\section{Sobrepeso/obesidad (IMC)}

Puede parecer lógico que los sujetos con sobrepeso y obesidad sean menos activos que los que se mantienen en el peso ideal, no obstante encontramos resultados contradictorios en las investigaciones que se han ido realizando cronológicamente.

Tiempo atrás se resaltaba que las personas que presentaban obesidad tendrían menor predisposición para participar en actividades físicas (Shephard y Cox, 1980). Sin embargo Ho (1987), consideraba que no existían datos relevantes que permitieran confirmar esta tendencia, debido a la carencia de relaciones significativas entre el exceso de peso y los practicantes del 
Flores, G.; Ruiz, F.; García, M.E. (2009). Relación de algunos correlatos biológicos y demográficos con la práctica físico-deportiva en estudiantes universitarios. El caso de la Universidad de Guadalajara, México.

ejercicio, después de concluir su estudio con adolescentes y jóvenes de 14 a 27 años en Hong Kong. En esta línea coincidían Adams-Campell, Washburn y Haile (1990) que, en un estudio con 192 estudiantes negros de primer año de la Universidad de Pittsburgh, examinaron algunos factores de riego para las enfermedades cardí deacas coronarias. Entre los resultados, descartaban cualquier relación entre los comportamientos de la práctica de actividad física y el Índice de Masa Corporal (IMC) en ambos sexos, disolviendo cualquier influencia del IMC en los niveles de actividad.

Estudios longitudinales (Schmitz, French y Jeffery, 1997) encuentran una asociación entre el Índice de Masa Corporal y la práctica de actividad física, ya que al producirse aumentos en el IMC de los sujetos, disminuyen los niveles de actividad y cuando se presentan disminuciones del IMC se incrementan los niveles de práctica. Resultando difícil encontrar evidencia que confirme esta noción en jóvenes y adultos (Sallis, 1999).

Miller, Staten y Rayens (2005), destacaban una relación entre los niveles de actividad física y el IMC de los sujetos. Esta asociación se produce cuando los estudiantes que realizan actividades físicas de nivel vigoroso perciben tener sobrepeso y obesidad, en tanto los que buscan cambiar el peso eligen actividades tanto vigorosas como las moderadas.

Estudios recientes revelan que los adolescentes con sobrepeso y obesidad demuestran una baja participación en la práctica deportiva con respecto a quienes tienen un peso ideal, manifestando una actitud menos positiva hacia la actividad física (Deforche, Bourdeaudhuij y Tanghe, 2006).

\section{Historial de lesiones y días de enfermedad}

Uno de los factores que probablemente conducen a los sujetos a disminuir la práctica de actividad física (Pollock, Gettman, Milesis, Bah, Durstine y Johnson, 1977), podría ser a causa de algunas lesiones que han sufrido como consecuencia de la participación en actividades físicas (Sallis, 1999).

Al respecto Sallis, Hovell, Hofstetter, Elder, Faucher, Spry, et al. (1990), examinaron qué factores estaban provocando la recaída de la población en la práctica del ejercicio, observando el historial de lesiones que habían sufrido a lo largo de su vida 2.043 sujetos, residentes de San Diego (EE.UU.). Entre los resultados encontraron que las personas que manifestaron haber sufrido lesiones a lo largo de su vida, habían limitado su participación en la práctica de actividad física, por ello, sufrir comúnmente alguna lesión conduce a disminuciones y probablemente al abandono de la práctica.

También es cierto que los individuos que suelen ser activos durante el tiempo libre, tienen más probabilidad de presentar algunos episodios de lesión (Carlson, Hootman, Powell, Macera, Heath, Gilchrist, et al., 2006; Plotnikoff, Mayhew, Birkett, Loucaides y Fodor, 2004), siendo los más vulnerables los jóvenes de 18 a 24 años.

Para Sallis et al. (1990), Schmitz, Lytle, Phillps, Murray, Birnbaum y Kubik (2002) y Schneider, Seither, Tonges y Zimmermann-Stenzel (2006), las lesiones también significan barreras que llevan a disminuir la participación en la práctica físico-deportiva, al presentarse el temor de volver a sufrir alguna lesión que afecte su salud, siendo más común en mujeres y conforme aumenta la edad de los sujetos. 
Flores, G.; Ruiz, F.; García, M.E. (2009). Relación de algunos correlatos biológicos y demográficos con la práctica físico-deportiva en estudiantes universitarios. El caso de la Universidad de Guadalajara, México.

\section{La actividad físico-deportiva en poblaciones universitarias}

En contraste con los estudios publicados, observamos cómo algunos correlatos producen cambios notables en la fuerza y dirección de asociación positiva y negativa en la práctica físico-deportiva. Supone observar la asociación o correlación que produce en los tres estados de la práctica en el alumnado universitario. Es decir, entre quienes nunca han practicado, los que habiendo sido activos han dejado de participar y los que se consideran practicantes.

Haciendo un análisis cronológico en diversas poblaciones, resulta sorprendente la variación de práctica entre los jóvenes universitarios.

Pinto y Marcus (1995), desarrollaron un estudio para conocer el comportamiento de 800 jóvenes respecto a la participación en actividades físicas y deportivas. Observaron que solo un poco más de la mitad de los estudiantes de una Universidad privada de Rhode Island (54\%), participaban en alguna actividad física o deportiva, en contraste con quienes no practicaban. Mostrando preocupación por el alto índice de inactividad dadas las repercusiones negativas que genera no realizar actividades físicas.

En tanto Blasco, Capdevila, Pintanel, Valiente y Cruz (1996), mostraban que el 74\% de los jóvenes dedicaban al menos una hora semanal a la práctica, en contraste con quienes afirmaban no dedicar ninguna. Evidenciando la escasa o insuficiente participación del alumnado de primer curso de la Facultad de Ciencias y Psicología de la Universidad Autónoma de Barcelona, después de haber sido clasificados como activos o inactivos a partir de las horas que destinaban a la práctica de actividad físico-deportiva durante la semana.

Ahora bien, parece que en otras poblaciones la diferencia entre activos e inactivos resultan más amplías, mostrando un mayor porcentaje de practicantes respecto a los no practicantes. Sánchez Pérez et al. (1998), indagaron sobre la participación de lo jóvenes universitarios de Deusto en actividades físico-deportivas. Tras analizar la muestra seleccionada (273) pertenecientes a las licenciaturas de Psicología, Informática, Pedagogía, Derecho y Diplomatura de Educación Social, encontraron que aproximadamente seis de cada diez estudiantes $(64 \%)$ practican actividades físicas, en relación con los inactivos $(35,4 \%)$.

Parecidos resultados en cuanto a las cifras de practicantes los obtuvo Leslie et al. (1999) en la Deakin University en Australia, observando que el 60\% de los estudiantes participaba en actividades físicas de tiempo libre, en contraste con quienes no lo hacían (40\%). Estas cifras fueron observadas luego de categorizar a 2.729 estudiantes como activos o inactivos de acuerdo a la actividad física que realizaban según las estimaciones producidas por los gastos energéticos (kcal/semana).

En cambio, existen datos que resultan contradictorios a los observados hasta el momento. Donde Figueiredo y Rebollo (2003), que analizaron los hábitos deportivos de los estudiantes brasileños de la Enseñanza Técnica CEFET de Paraíba, reportaban que apenas el 34\% de los alumnos practicaba en el momento del estudio actividades físicas, seguido de un $60 \%$ que había abandonado la práctica y un $6 \%$ que nunca la había practicado. En otras palabras, aproximadamente dos de cada tres estudiantes no realizaban práctica de actividad física.

Continuando con estudios en el ámbito universitario, se aprecia, con respecto a las tasas de practicantes, que en la investigación desarrollada en la corporación universitaria Lasallista en Colombia (Rodríguez, Castañeda, Correa y Sarabia, 2004), se ha podido apreciar que, poco más de la mitad de los estudiantes de esta comunidad universitaria son practicantes $(52 \%)$, en contraste con quienes no lo son. 
Flores, G.; Ruiz, F.; García, M.E. (2009). Relación de algunos correlatos biológicos y demográficos con la práctica físico-deportiva en estudiantes universitarios. El caso de la Universidad de Guadalajara, México.

Revista Internacional de Ciencias del Deporte. 14(5), 59-80. http://www.cafyd.com/REVISTA/01406.pdf

Si nos centramos en la investigación llevada a cabo en la Universidad de Almería (Ruiz Juan et al., 2005) en el curso académico 2000-2001, se observa el 38,6\% de los encuestados de primer ciclo, realiza actividades físico-deportivas en el momento de realización de la investigación, seguido de un 47,7\% que había tenido que abandonarla, y un 13,6\% que nunca había practicado. Con respecto al segundo ciclo, las cifras en los practicantes $(35,6 \%)$ son menores que los de primer ciclo, situación similar que se presenta en quienes la han abandonado $(40,8 \%)$ produciendo un aumento en los que nunca $(23,6 \%)$ han practicado.

\section{Problema}

Según cifras del Instituto Nacional de Estadística Geografía e Informática (2006) los jóvenes mexicanos de entre 15 y 29 años representan el $28 \%$ de la nación donde el $52 \%$ son mujeres y el resto hombres (48\%). Estos sectores de la población, según datos de la Organización Mundial de la Salud (OMS) (1998), comienzan a sufrir algunas enfermedades que años atrás afectaban principalmente a los sujetos en edad adulta (enfermedades del corazón, tumores malignos, diabetes mellitus, accidentes, insuficiencia renal, etc.) y han pasado a ser causa del $77 \%$ de las muertes en chicos y del 56\% en chicas (INEGI, 2003).

En México, escasean estudios sobre los comportamientos de los jóvenes universitarios con respecto a su participación en la actividad físico-deportiva de tiempo libre, destacando los trabajos de Márquez Ceniceros, Delgado Fernández y López Walle (2003). Lo que hace necesario mirar hacia otras poblaciones universitarias del territorio nacional.

Considerando las repercusiones positivas y negativas que trae consigo la actividad e inactividad física en la salud de las personas, y dado el incremento en la aparición de enfermedades que afectan a la población joven en el mundo, particularmente a los mexicanos, consideramos necesario indagar qué factores, particularmente algunos biológicos y demográficos (sexo, edad, IMC, lesiones y días de enfermedad), se asocian con la adherencia y no adherencia de participación en actividades físicas y deportivas de tiempo libre. Particularmente en los estudiantes de Educación Superior de la Universidad de Guadalajara, México.

A partir de estos planteamientos y lo observado en el marco teórico, nos cuestionamos:

o ¿Son suficientemente activos los estudiantes de la Universidad de Guadalajara como para lograr beneficios en su salud y reducir la aparición de algunas enfermedades que afectan a estos sectores?

o ¿Puede ser que la implicación en la práctica de actividad físico-deportiva de tiempo libre, de hombres y mujeres en nuestra población, presente diferencias importantes como muestran el resto de los estudios?

o ¿Serán los estudiantes universitarios de mayor edad, con respecto a los más jóvenes, quienes más declinan en la práctica?

o ¿El haber sufrido alguna lesión o días de enfermedad serán causas que conduzcan a los estudiantes a disminuir la práctica de actividad físico-deportiva de tiempo libre?

o ¿Será cierto que no tener un peso ideal conlleva a los jóvenes a ser menos participativos en las actividades físicas de tiempo libre, respecto a los que sí lo tienen, o por lo contrario no existe relación? 
Flores, G.; Ruiz, F.; García, M.E. (2009). Relación de algunos correlatos biológicos y demográficos con la práctica físico-deportiva en estudiantes universitarios. El caso de la Universidad de Guadalajara, México.

\section{Hipótesis}

Será mayor la proporción de estudiantes que realiza práctica respecto a quienes han abandonado o nunca han practicado.

$>$ Las mujeres son el colectivo que en menor proporción participa en actividades físicodeportivas.

$>$ El alumnado universitario de mayor edad, es más probable en declinar en la práctica.

$>$ Quienes comúnmente sufren lesiones, son más propensos en declinar en la participación en actividades físicas.

$>$ Los estudiantes con peso normal son más participativos, respecto a quienes no cuentan con dicho peso.

Por tanto en este estudio nos planteamos los siguientes dos objetivos. Primeramente, estimar las tasas de sujetos que nunca han practicado actividades físico-deportivas de tiempo libre, los que habiendo sido activos han tenido que abandonar y los que son practicantes en activo. El segundo, consiste en analizar entre algunos factores biológicos y demográficos (sexo, edad, IMC, lesiones y días de enfermedad) cuales se correlacionan en mayor o menor medida con los comportamientos de práctica físico-deportiva de tiempo libre.

\section{Metodología}

\section{Población y muestra}

La población objeto de estudio la constituyen los estudiantes de Educación Superior de la Universidad de Guadalajara (México), durante el ciclo escolar 2005 calendario "B", distribuidos en los catorce centros de la red Universitaria. Para la obtención de la información sobre la población objetivo de estudio hemos tomado los datos publicados por la coordinación de control escolar, cuya población está constituida por 65.690 sujetos, de los cuales 33.537 son hombres y 32.163 las mujeres (Universidad de Guadalajara, 2004).

Para seleccionar la muestra se llevó a cabo un muestreo polietápico estratificado con afijación proporcional. En primera instancia fue por Centro Universitario, a continuación se realizó de acuerdo al nivel de estudios (licenciatura y/o técnico superior universitario) y, por último, se consideró la variable sexo, respetando en cada uno de los estratos la misma fracción del muestreo.

Teniendo en cuenta los datos de la población objeto de estudio hemos buscado que la validez de los resultados nos permita asumir un margen de error de $\pm 3 \%$ y un nivel de confianza del $95,5 \%$. Es decir, la muestra seleccionada para nuestro análisis es de 1.207 estudiantes universitarios, de los cuales 614 son hombres y 593 mujeres (tabla 1). 
Flores, G.; Ruiz, F.; García, M.E. (2009). Relación de algunos correlatos biológicos y demográficos con la práctica físico-deportiva en estudiantes universitarios. El caso de la Universidad de Guadalajara, México.

Revista Internacional de Ciencias del Deporte. 14(5), 59-80. http://www.cafyd.com/REVISTA/01406.pdf

Tabla 1. Afijación de la muestra del alumnado de la Universidad de Guadalajara (2004), por centro universitario.

\begin{tabular}{|c|c|c|c|c|c|c|}
\hline \multirow{2}{*}{ Centro Universitario } & \multicolumn{3}{|c|}{ Población (N) } & \multicolumn{3}{|c|}{ Muestra (n) } \\
\hline & Hombre & Mujer & Total & Hombre & Mujer & total \\
\hline $\begin{array}{l}\text { Centro Universitario de } \\
\text { Arte, Arquitectura y Diseño }\end{array}$ & 2.594 & 2.238 & 4.832 & 45 & 38 & 83 \\
\hline $\begin{array}{c}\text { Centro Universitario de } \\
\text { Ciencias Biológicas y Agropecuarias }\end{array}$ & 1.417 & 1.170 & 2.587 & 24 & 19 & 44 \\
\hline $\begin{array}{c}\text { Centro Universitario de } \\
\text { Ciencias Económicas y Administrativas }\end{array}$ & 5.600 & 7.840 & 13.440 & 93 & 131 & 225 \\
\hline $\begin{array}{c}\text { Centro Universitario de } \\
\text { Ciencias Exactas e Ingenierías }\end{array}$ & 8.989 & 2.904 & 11.893 & 151 & 51 & 202 \\
\hline $\begin{array}{l}\text { Centro Universitario de } \\
\text { Ciencias de la Salud }\end{array}$ & 2.496 & 4.102 & 6.598 & 42 & 70 & 112 \\
\hline $\begin{array}{c}\text { Centro Universitario de } \\
\text { Ciencias Sociales y Humanidades }\end{array}$ & 3.417 & 4.185 & 7.602 & 60 & 68 & 128 \\
\hline $\begin{array}{l}\text { Centro Universitario } \\
\text { de los Altos }\end{array}$ & 698 & 863 & 1.561 & 18 & 20 & 38 \\
\hline $\begin{array}{c}\text { Centro Universitario } \\
\text { de la Ciénega }\end{array}$ & 2.083 & 2.185 & 4.268 & 44 & 49 & 93 \\
\hline $\begin{array}{c}\text { Centro Universitario } \\
\text { de la Costa }\end{array}$ & 2.153 & 2.282 & 4.435 & 47 & 48 & 95 \\
\hline $\begin{array}{l}\text { Centro Universitario } \\
\text { de la Costa Sur }\end{array}$ & 1.208 & 1.053 & 2.261 & 28 & 23 & 51 \\
\hline $\begin{array}{l}\text { Centro Universitario } \\
\text { de Lagos de Moreno }\end{array}$ & 837 & 727 & 1.564 & 18 & 17 & 35 \\
\hline $\begin{array}{c}\text { Centro Universitario } \\
\text { del Norte }\end{array}$ & 259 & 405 & 664 & 5 & 9 & 14 \\
\hline $\begin{array}{l}\text { Centro Universitario } \\
\text { del Sur }\end{array}$ & 1.121 & 1.557 & 2.678 & 24 & 35 & 58 \\
\hline $\begin{array}{l}\text { Centro Universitario } \\
\text { de los Valles }\end{array}$ & 665 & 642 & 1.307 & 14 & 15 & 29 \\
\hline Total & 33.537 & 32.153 & 65.690 & 614 & 593 & 1.207 \\
\hline
\end{tabular}


Flores, G.; Ruiz, F.; García, M.E. (2009). Relación de algunos correlatos biológicos y demográficos con la práctica físico-deportiva en estudiantes universitarios. El caso de la Universidad de Guadalajara, México.

Revista Internacional de Ciencias del Deporte. 14(5), 59-80. http://www.cafyd.com/REVISTA/01406.pdf

\section{Material y método}

Dadas las características propias de la investigación, el instrumento manejado ha sido el cuestionario. Para la elaboración del cuestionario fue necesaria la delimitación del campo de estudio y determinación precisa de los objetivos que pretendemos lograr en nuestra investigación, basándonos en algunos estudios con similares características a la nuestra (Biddle Gorey y Stensel, 2004; IPAQ, 2002; Leslie et al., 2001; Ruiz Juan et al., 2001; Ruiz Juan y García Montes, 2005; Ruiz Juan et al., 2005 y Sallis, Hovell y Hofstetter, 1992), en diferentes campos (metodología, sociología, y del ámbito de las ciencias de la actividad físico-deportiva).

Mediante un total de 66 preguntas, fueron explorados los siguientes bloques: 1) factores biológicos y demográficos, 2) factores psicológicos, cognitivos y emocionales, 3) hábitos saludables, 4) factores sociales y culturales, 5) características de las actividades físicas y deportivas e 6) índice de actividad física en la vida cotidiana (IPAQ).

Para el presente artículo se seleccionaron del cuestionario las siguientes variables: comportamientos de los estudiantes universitarios respecto a la práctica físico-deportiva de tiempo libre, y algunos de los factores biológicos y demográficos como el sexo, la edad, el sobrepesoobesidad y los días de enfermedad o lesión.

La validación del cuestionario se realizó por medio de la validez de contenido y de constructo, puesto que el objetivo del cuestionario es medir conductas, comportamientos y opiniones expresadas por cada uno de los sujetos de la población objeto de estudio. La validez del contenido hace referencia al examen del contenido del cuestionario para determinar si son una muestra relevante y representativa del objetivo que se pretende medir. La validez del constructo es un concepto hipotético que forma parte de las teorías que se intentan explicar y que se utiliza para agrupar bajo una etiqueta de un constructo una conducta particular (Buendía, 1994).

La constatación de la fiabilidad del cuestionario se logró a través de los tres diferentes estudios pilotos que hemos realizado en la ciudad de Guadalajara, México, con la finalidad de probar si el contenido de las preguntas, terminología y vocabulario eran entendidos por los entrevistados. Esto permitió hacer correcciones, para perfeccionar y dominar el contenido del cuestionario, destacando las diferentes aportaciones proporcionadas por destacados especialistas y expertos consultados.

\section{Procedimiento y análisis de los datos}

El trabajo de campo se realizó en los meses de octubre a diciembre de 2005, en los centros temáticos y regionales de la red universitaria, aplicándose en aulas de manera autoadministrada en presencia de un encuestador. Destinando el alumno un promedio de 20 minutos a su cumplimentación

Para el análisis de los datos utilizamos el programa informático Statistickal Package for Social Sciences (SPSS/PC versión 14.0) que posibilita la puesta en práctica de las técnicas de análisis precisas en este estudio: análisis descriptivo y análisis inferencial.

La estadística descriptiva se realizó a través de tablas de frecuencia y porcentaje. El análisis inferencial, consistente en establecer el grado de relación y asociación entre variables, se realizó a través de tablas de contingencias (muestran frecuencia y porcentajes de las respuestas, distribuidas en función de las variables asociadas) en las que se establece el grado de significación (NS) del contraste de la prueba de Chi-cuadrado de Pearson $\left(\chi^{2}\right)$ entre variables. Si la 
Flores, G.; Ruiz, F.; García, M.E. (2009). Relación de algunos correlatos biológicos y demográficos con la práctica físico-deportiva en estudiantes universitarios. El caso de la Universidad de Guadalajara, México.

prueba de $\chi^{2}$ es mayor que 0,05 hay una independencia entre las variables. Si por el contrario, es menor a esta cifra, se afirma que hay asociación entre las variables. Para considerar el valor Chi-cuadrado de Pearson se constató que se cumplían las condiciones de validez, es decir, que no hubiese ninguna casilla con frecuencia esperada menor que uno y que no más del $20 \%$ de ellas tuvieran frecuencia esperada menor que cinco.

\section{Resultados}

\section{Descripción la población estudiada}

\section{$\underline{\operatorname{SexO}}$}

En lo que refiere al sexo, la población estudiada se encuentra bastante equilibrada, observándose una ligera inclinación que se manifiesta en menos de dos puntos del valor porcentual a favor de los hombres. Es decir, el 50,8\% de la muestra son hombres mientras que el resto son mujeres $(49,2 \%)$ (figura 1$)$.

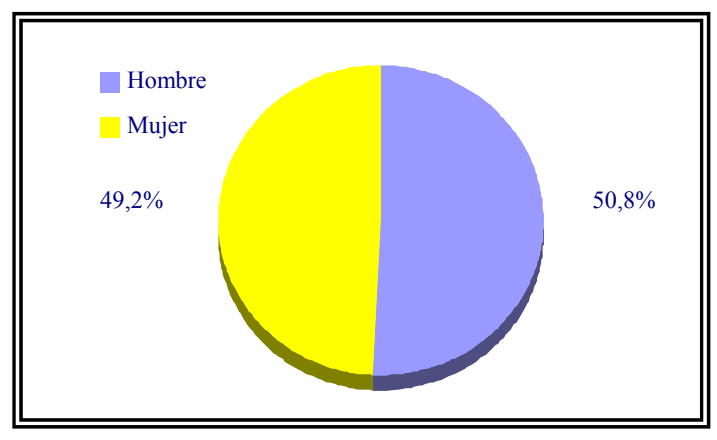

Fig. 1. Sexo del alumnado universitario

\section{$\underline{\text { Edad }}$}

Dadas las características de la población estudiantil, cuya media de edad oscila entre los 20 años, se formaron tres grupos para su identificación. El grupo de edad hasta los 20 años constituye el mayor conglomerado dentro de la muestra, con un porcentaje del 48,6\% teniendo valores porcentuales superiores, en varios puntos, a los demás grupos. En cuanto al grupo de más de 26 años, se caracteriza porque su proporción $(8,1 \%)$ es la menor. Entre ambos grupos se ubican los estudiantes de 21 a 25 años como el segundo mayor valor $(43,3 \%)$ (figura 2 ).

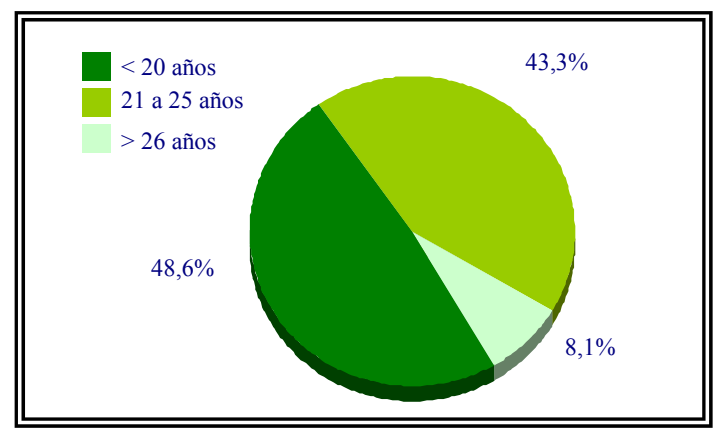

Figura 2. Distribución de grupos de edad del alumnado universitario 
Flores, G.; Ruiz, F.; García, M.E. (2009). Relación de algunos correlatos biológicos y demográficos con la práctica físico-deportiva en estudiantes universitarios. El caso de la Universidad de Guadalajara, México.

\section{Obesidad/sobrepeso (IMC)}

Para tener un indicador significativo que se aproxime a los posibles problemas relacionados con el peso (antropométrico) entre los estudiantes, se ha recurrido a utilizar como herramienta de investigación el Índice de Masa Corporal (IMC), que se calcula con la variable peso y altura de la persona.

El IMC de la muestra seleccionada ha sido determinado según la fórmula: peso/altura ${ }^{2}$ (USDHHS, 2006). Posteriormente, se formaron cuatro grupos permitiendo clasificar a los sujetos para su identificación en las siguientes categorías:
* Insuficiente (menos de 18.5)
* Normal (entre 18.5 y 25)
* Sobrepeso (entre 25 y 30 )
* Obesidad (más de 30).

Según el análisis estadístico el IMC de los estudiantes es disparejo. Dos de las terceras partes $(66,6 \%)$ se encuentra dentro de los niveles de grasa recomendados, es decir, con un índice normal (entre 18.5 y 25 ). En cambio, un $20,4 \%$ de los sujetos comienzan a tener ciertos problemas de sobrepeso al encontrarse sus niveles de grasa entre 25 y 30 . Sobresale que un colectivo de jóvenes $(7,6 \%)$ mantiene un nivel insuficiente al situarse por debajo de 18.5 y el resto $(5,5 \%)$ presenta un estado de obesidad al situarse por encima de 30 (figura 3 ).

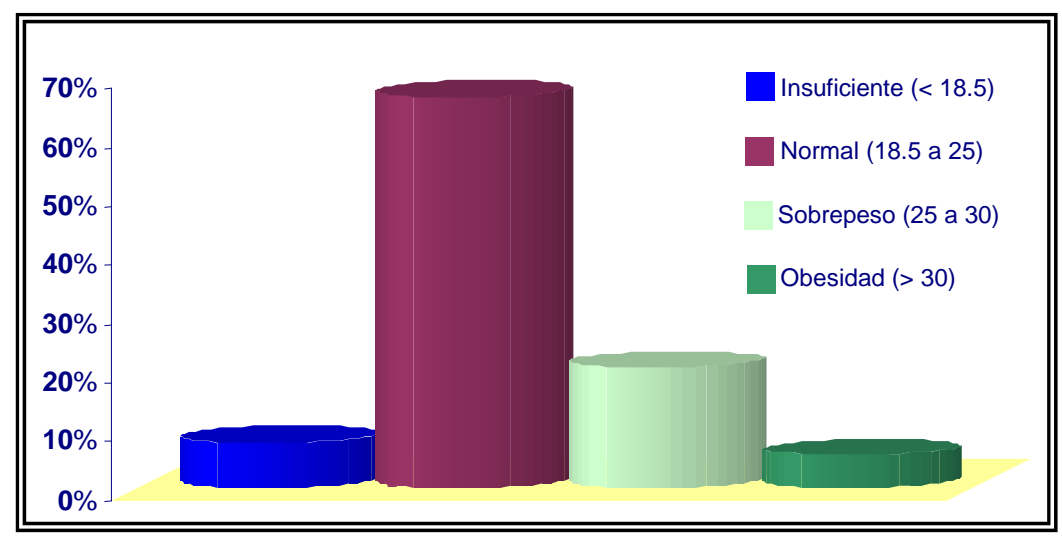

Figura 3. Índice de Masa Corporal en el alumnado de la Universidad de Guadalajara.

\section{Enfermedad o lesiones}

La población objeto de estudio es diversa con respecto a los días que se han encontrado enfermos o tenido algún tipo de lesión durante el transcurso del último año previo a la aplicación del cuestionario. Resulta bastante llamativo que entre los valores más elevados se ubique la categoría de los que manifiestan haber tenido un lapso menor a los siete días de enfermedad o lesión $(47,8 \%)$, es decir, un elevado porcentaje de alumnos cuando enferma o tiene alguna lesión suele ser durante muy pocos días. Quienes indican haberlo estado más de 30 días son el $13,7 \%$ y, entre ambos grupos, se localizan quienes de 7 a 15 días (22,7\%), 15 a 21 días (5,9\%) y 21 a 30 días $(5,9 \%)$ se ha encontrado enfermos o tenido alguna lesión durante los últimos doce meses (figura 4). 
Flores, G.; Ruiz, F.; García, M.E. (2009). Relación de algunos correlatos biológicos y demográficos con la práctica físico-deportiva en estudiantes universitarios. El caso de la Universidad de Guadalajara, México.

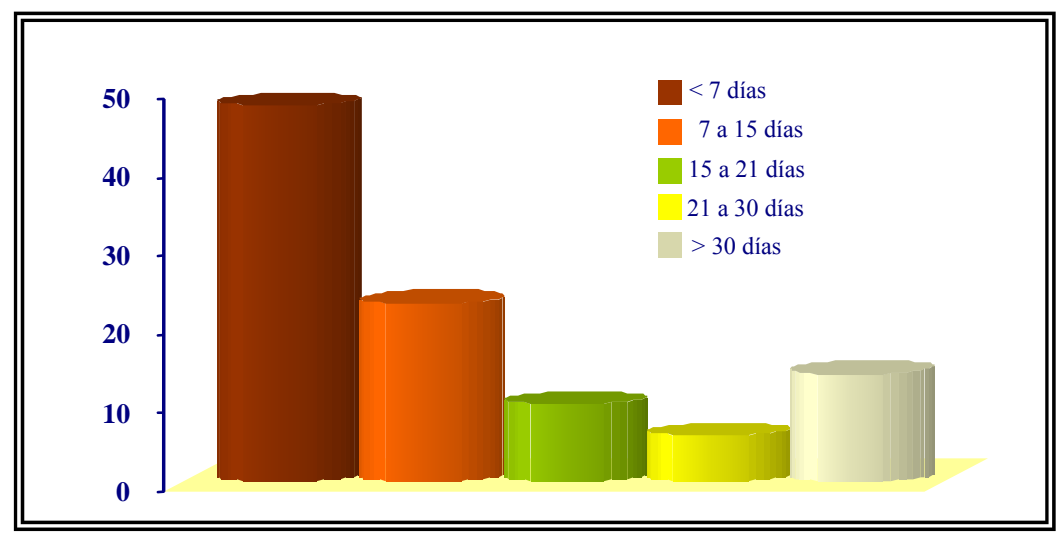

Figura 4. Días que han estado enfermos o han tenido algún tipo de lesión

Práctica físico-deportiva. Relación con el sexo, la edad, el IMC y los días de enfermedad o lesiones

Los datos permiten apreciar que la población estudiantil de la Universidad de Guadalajara, en su comportamiento con la práctica físico-deportiva, es diversa. Encontramos que el 56,8\% de los jóvenes se mantienen físicamente activos en la actualidad, mientras que el resto 43,2\% podríamos considerarlos como inactivos. Entre estos últimos, destaca un elevado porcentaje de estudiantes $(38,3 \%)$ que se han visto en la necesidad abandonar la práctica físico-deportiva durante su tiempo libre, al igual que sólo el 4,9\% nunca ha tenido la experiencia positiva de realizarla (figura 5).

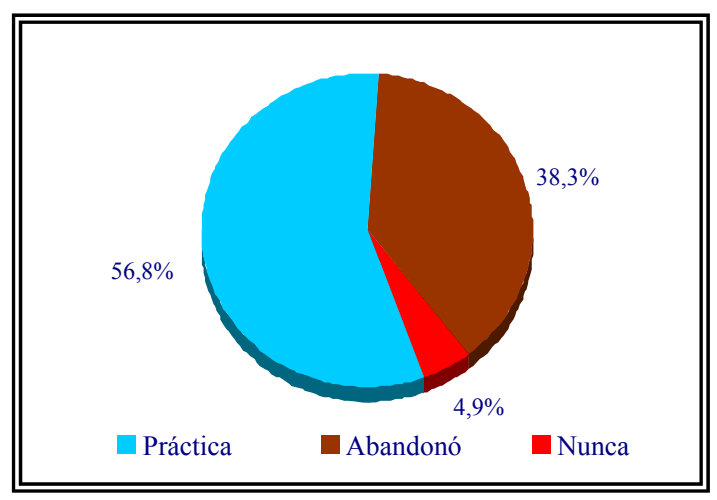

Figura 5. Experiencia del alumnado universitario respecto a la práctica físico-deportiva.

Una vez analizada esta descripción, nos planteamos la necesidad de verificar qué variables del grupo de factores biológicos y demográficos, podrían asociar o influir con la participación del alumnado de la Universidad de Guadalajara, en las actividades físico-deportivas de tiempo libre. En particular, nos centraremos en los correlatos que según la literatura, resultan más significativos de manera positiva o negativa en estos sectores de la población, tales como el sexo, la edad, el índice de masa corporal (IMC) y los días de enfermedad/lesiones. 
Flores, G.; Ruiz, F.; García, M.E. (2009). Relación de algunos correlatos biológicos y demográficos con la práctica físico-deportiva en estudiantes universitarios. El caso de la Universidad de Guadalajara, México.

En cuanto al sexo, los datos permiten observar que entre esta variable y la práctica físicodeportiva existe, de acuerdo al Chi-cuadrado de Pearson, una correlación al encontrarse diferencias estadísticamente significativas $(\mathrm{p}<, 000)$. Con respecto a los que dicen nunca haber realizado y abandonado la práctica físico-deportiva, son las mujeres quienes presentan los índices más elevados de inactividad en comparación con los hombres $(7,1 \%$ frente al $2,8 \%$, en nunca han realizado y $44,9 \%$ frente a $31,9 \%$ en abandono). En cuanto a mantenerse físicamente activo son los hombres $(65,3 \%)$ quienes suelen ser más participativos que las mujeres (48\%). Por lo tanto, podemos reseñar que las mujeres son más inactivas que los hombres cuando nos referimos a la participación en actividades físico-deportivas de tiempo libre (tabla 2 y figura 6 ).

Tabla 2. Sexo del alumnado universitario en los comportamientos de actividad físico-deportiva

\begin{tabular}{|cccc|}
\hline & \multicolumn{3}{c|}{ Sexo } \\
& Hombre & Mujer & Total \\
Nunca he practicado & 2,8 & 7,1 & 4,9 \\
La he abandonado & 31,9 & 44,9 & 38,3 \\
Realizo práctica & 65,3 & 48,0 & 56,8 \\
\hline
\end{tabular}

Chi-cuadrado de Pearson $\mathrm{p}<, 000$

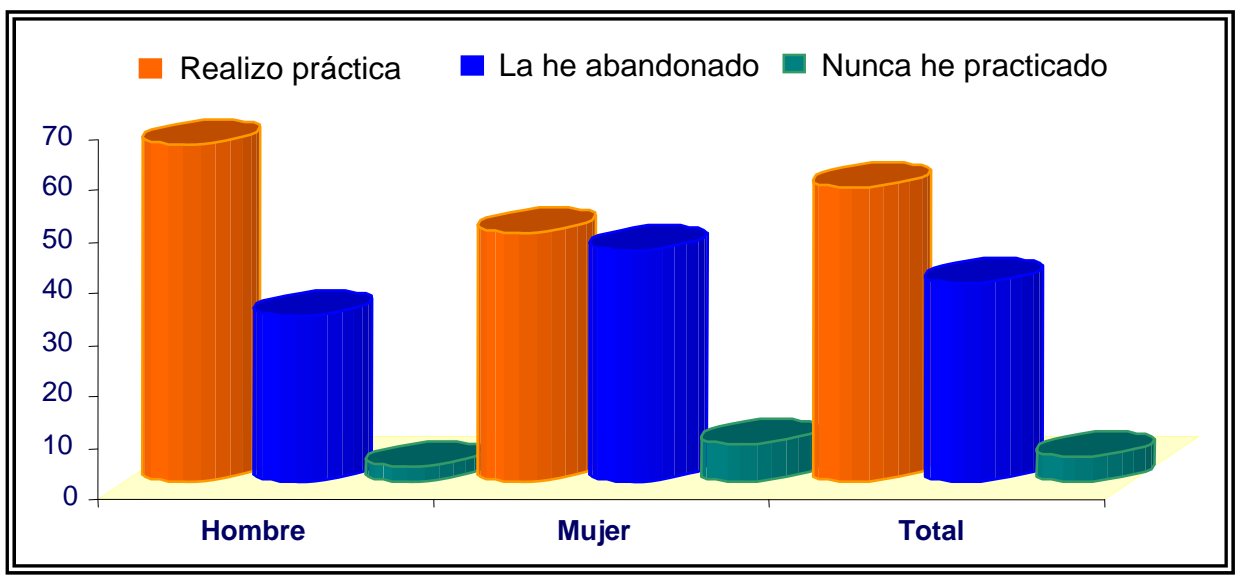

Figura 6. Sexo del alumnado universitario en los comportamientos de actividad físico-deportiva

Con respecto a la edad no se produce correlación alguna entre esta variable y la práctica físico-deportiva, pues las estadísticas revelan que no existen diferencias significativas $(p<, 304)$. Es decir, que el hecho de ser más o menos joven no condiciona, en esta población, la participación en la práctica físico-deportiva de tiempo libre ya que los resultados son similares a la media poblacional.

En lo que refiere al IMC, al analizar la correlación entre esta variable y la práctica físicodeportiva de tiempo libre se constata la inexistencia de correlación, ya que el Chi-cuadrado de Pearson $(\mathrm{p}<, 200)$ indica que no hay diferencias significativas. Es decir, que el IMC que tengan los alumnos de la Universidad de Guadalajara no correlaciona en la disponibilidad de participación en actividades físicas y deportivas. 
Flores, G.; Ruiz, F.; García, M.E. (2009). Relación de algunos correlatos biológicos y demográficos con la práctica físico-deportiva en estudiantes universitarios. El caso de la Universidad de Guadalajara, México.

Revista Internacional de Ciencias del Deporte. 14(5), 59-80. http://www.cafyd.com/REVISTA/01406.pdf

Por último, en cuanto a los días que han estado enfermos o tenido algún tipo de lesión durante los últimos doce meses, se descarta cualquier correlación de las variables $(p<, 135)$. Por lo tanto, haber estado enfermo o lesionado no es una variable que afecte o favorezca la adherencia o no a la práctica físico-deportiva.

\section{Discusión de los resultados}

\section{La práctica de actividad físico-deportiva en los estudiantes de educación superior de la Uni- versidad de Guadalajara}

Basta analizar la literatura científica, para percatarse sobre la latente preocupación que existe sobre la promoción de la práctica de actividad física y el deporte, dado los beneficios positivos que son atribules a esta y, las repercusiones negativas que fomenta la inactividad. Esta valoración ha dado cabida a los investigadores a estimar qué cantidad y calidad debe tener la actividad física, cuando es realizada por los sujetos para lograr beneficios para su salud y reducir la aparición de enfermedades (Pate et al., 1995; Pollock et al., 1998; USDHHS, 1996 y 2000). Por lo cual se hace necesario identificar qué sectores de la población joven resultan activos o inactivos en la práctica, con el fin de realizar eficaces intervenciones para promocionar la adherencia y continuidad de la misma.

Llama la atención que dentro de los estudios se hace mucho hincapié sobre los sujetos inactivos, debido a las consecuencias negativas que para estos puede acarrear en un futuro no muy lejano. Se consideran sujetos inactivos, por una parte, los que por diferentes circunstancias nunca han participado en actividades físico-deportivas en su tiempo libre $\mathrm{y}$, por otra parte, los que habiendo sido activos han abandonado la práctica por diversas circunstancias.

En nuestra investigación, el colectivo de los que nunca han practicado está representado, afortunadamente, por un porcentaje muy bajo, coincidiendo con otros estudios de ámbito universitario (Figueiredo y Rebollo, 2003; Ruiz Juan et al., 2005 y Sánchez Pérez et al., 1998). Evidenciando que aunque las cifras son bajas, los datos son alarmantes en poblaciones tan jóvenes, dada la importancia que existe en las sociedades avanzadas por el impulso de la práctica que permita llevar un estilo de vida activo. Resultando comprensible el porqué los jóvenes comienzan a sufrir algunas enfermedades que años atrás afectaban particularmente a la edad adulta (OMS, 1998), debido a la inactividad física.

Respecto al otro conjunto de estudiantes inactivos, llama especialmente la atención que las cifras se disparan, habiendo abandonado, aproximadamente, cuatro de cada nueve que eran activos, reforzando lo expuesto anteriormente sobre las particularidades de esta etapa universitaria. Llama la atención, haciendo un análisis comparativo en cuanto a las cifras, que nuestros datos coinciden con el promedio de universitarios que han abandonado la práctica en Deusto (Sánchez Pérez et al., 1998), en el supuesto de que las diferencias económicas, políticas y sociales entre mexicanos y españoles deben ser consideradas, además de las discrepancias en la fechas entre los estudios. Sin embargo, aportaciones recientes (Figueiredo y Rebo1lo, 2003; Ruiz Juan et al. 2005), presentan porcentajes superiores de abandono. De esta manera y coincidiendo con Kilpatrick et al. (2005), es preocupante la disminución en la participación de la juventud universitaria en la práctica físico-deportiva. Lo cual es un grave problema general y no solamente de poblaciones universitarias en particular. 
Flores, G.; Ruiz, F.; García, M.E. (2009). Relación de algunos correlatos biológicos y demográficos con la práctica físico-deportiva en estudiantes universitarios. El caso de la Universidad de Guadalajara, México.

Revista Internacional de Ciencias del Deporte. 14(5), 59-80. http://www.cafyd.com/REVISTA/01406.pdf

Para Márquez et al. (2006), OMS (2002) y USDHHS (1996), los sujetos que realizan y mantienen su participación en la actividad físico-deportiva disminuyen de forma considerable la aparición de algunos padecimientos crónicos o muerte prematura. Siguiendo estos comentarios podríamos considerar que en nuestro caso, poco más de la mitad del alumnado podría estar recibiendo algunos beneficios saludables, puesto que en el momento del estudio manifestaron realizar actividades físico-deportivas en su tiempo libre. Tendencia que coincide con otras investigaciones (Pinto y Marcus, 1995; Rodríguez et al., 2004; Ruiz Juan et al., 2005), cuyos resultados presentan similitud con las tasas de practicantes, pero difiere de otras (Figueiredo y Rebollo, 2003), cuyos resultados son adversos por las bajas tasas presentadas y de Blasco et al. (1996), Leslie et al. (1999) y Sánchez Pérez et al. (1998), porque sus estudiantes universitarios presentan mayores porcentajes de participación.

Poniendo de manifiesto que las tasas de practicantes presentan variaciones entre las diversas poblaciones universitarias. Es decir, podríamos pronosticar que en estos sectores de la población, la proporción de sujetos que participa en actividades físico-deportivas oscila entre un $30 \%$ y $70 \%$. Resultando provechoso para futuras investigaciones, indagar si la práctica realizada es suficiente y provechosa para cuantificar qué proporción de la población estudiantil, efectivamente podría lograr beneficios, y por otro lado, la que por su escasa práctica se encuentra en riego.

\section{Correlatos asociados con la práctica de actividad físico-deportiva}

Después de valorar la situación en que se encuentran los universitarios jaliscienses activos e inactivos, surgen algunas interrogantes que conducen a reflexionar que no bastan solo cifras, sino que es necesario indagar qué posibles causas podrían inducir a la continuidad o abandono de la práctica, ya que según observamos, puede ser afectada o favorecida por correlación de algunas variables que pueden condicionar la adherencia, continuidad y participación de las personas (Dishman y Sallis, 1994; Dishman et al., 1985; Sallis y Owen, 1999; Trost et al., 2002). Los investigadores han identificado una amplia gama de factores, resultando muy extenso realizar un análisis sobre cada uno.

Por ello indagamos, siguiendo a Sallis (1999), algunas variables biológicas y demográficas, que podrían según la literatura presentar alguna posible correlación con la participación o no del alumnado universitario en las actividades físico-deportivas de tiempo libre. Teniendo en cuenta que, aunque varios son los correlatos que integran este grupo, no todos han presentado asociaciones positivas o negativas con la participación o no en los jóvenes, siendo más probables en otras poblaciones.

Razón que hace necesario discutir con otros estudios qué correlación existe con el sexo, la edad, el IMC y las lesiones o días de enfermedad que pudieran causar variaciones en los índices de la práctica.

Los estudios en su mayoría ponen de manifiesto que los hombres son el colectivo que en mayor medida realiza práctica de actividades físico-deportiva durante su tiempo libre (Blasco et al., 1996; Hernández Rodríguez, 2001; Kelly y Sharpe, 1994; Leslie et al., 1999 ; Ruiz Juan et al., 2001; Ruiz Juan et al., 2005; Sánchez Pérez et al., 1998), resultando afirmativo en nuestro caso. Sin embargo, no puede afirmarse que las mujeres resultan ser menos participativas en todos los casos, dado que según observamos Von Bothmer y Fridlun (2005) no encuentran diferencias significativas entre hombres y mujeres universitarios del sudeste de Suecia. Pero, 
Flores, G.; Ruiz, F.; García, M.E. (2009). Relación de algunos correlatos biológicos y demográficos con la práctica físico-deportiva en estudiantes universitarios. El caso de la Universidad de Guadalajara, México.

Revista Internacional de Ciencias del Deporte. 14(5), 59-80. http://www.cafyd.com/REVISTA/01406.pdf

confirma la tendencia de que el sexo es una variable potente que debemos considerar en la promoción de la actividad física y el deporte.

Respecto a los valores porcentuales, resulta gratificante que las diferencias entre los alumnos y alumnas jaliscienses se encuentran entre las más bajas y las cantidades de practicantes superan, en ambos sexos, la mitad de la población.

Observamos que, con el paso del tiempo (atendiendo a la fecha de las diversas investigaciones referenciadas), no se producen modificaciones sustanciales que alteren la tendencia y equiparen las cifras de práctica de ambos colectivos, situación que preocupa y llama la atención, porque algo está ocurriendo desde tiempo atrás, que no ha permitido revocarla.

Hacer referencia nuevamente, tal como se ha expuesto en el marco teórico, que en el estudio de Ruiz Juan et al. (2005), al analizar la evolución producida en relación a las dos investigaciones realizadas anteriormente con los universitarios almerienses (Ruiz Juan et al., 2001 y Hernández Rodríguez, 2001), sí se aprecia un acercamiento en los porcentajes de los practicantes de ambos sexos, al mantenerse, afortunadamente, el de las chicas y descender, desgraciadamente, el de los chicos. Esto debe hacernos reflexionar, por un lado, sobre las características de las ofertas existentes y las oportunidades para unas y otros y, por otro lado, sobre las actuaciones que deben acometerse para evitar los abandonos e incrementar la vida activa de todos los jóvenes.

En cuanto a la variable edad, autores como Sallis (2000), señalan que cuando los sujetos son más jóvenes, aumentan las probabilidades de ser más activos, en otras palabras, conforme transcurren los años, estos decaen en la práctica. Situación que según Keating et al. (2005) resulta más fácil observar esto en poblaciones escolares (niños y adolescentes) que en universitarias, debido a que, en estas últimas, existe mayor diversidad en los grupos de edad, dificultando realizar estimaciones con otros estudios.

En nuestro caso, no se presentan resultados estadísticamente significativos atendiendo a los diferentes grupos de edad planteados entre los universitarios jaliscienses, por lo que, aunque las datos muestren que los estudiantes mayores de 21 años resultan ser más activos que los de menor edad, no podemos afirmar que difieren de la teoría de Sallis (2000) y podrían inclinarse hacia la de Keating et al. (2005). Pero, los estudios realizados por Hernández Rodríguez (2001), Ruiz Juan (2000) y Ruiz Juan et al. (2005), muestran que con el paso del tiempo el índice de practicantes, en los mismos grupos de edad, pero diferentes sujetos, no presentan tendencias que ratifiquen que los más jóvenes, resulten ser más activos. Esto hace suponer que los más jóvenes podrían disminuir la práctica en la transición del bachillerato a la universidad y en los primeros semestres en la misma, ocasionado por los múltiples cambios que enfrentan al ingresar en esta nueva etapa de los estructurados y guiados periodos escolares previos.

Pasando a analizar el IMC, los resultados reflejan que en la población universitaria jalisciense, tener el peso ideal o excederse del mismo, no produce variaciones en la práctica de actividad físico-deportiva de tiempo libre. Datos que coinciden a los difundidos por AdamsCampell et al. (1990) y Ho (1987), siendo contradictorios a los de Deforche et al. (2006) y Schmitz et al. (1997) que encuentran asociaciones entre la disminución de la práctica en los sujetos y su peso. Independientemente de las contradicciones existentes entre unos y otros estudios, debemos hacer una llamada de atención sobre la relación obesidad y salud y, la posible incidencia en la disminución de práctica de actividad físico-deportiva, hecho que es sufi- 
Flores, G.; Ruiz, F.; García, M.E. (2009). Relación de algunos correlatos biológicos y demográficos con la práctica físico-deportiva en estudiantes universitarios. El caso de la Universidad de Guadalajara, México.

Revista Internacional de Ciencias del Deporte. 14(5), 59-80. http://www.cafyd.com/REVISTA/01406.pdf

cientemente relevante para que las autoridades sociales, políticas y universitarias afronten el tema para evitar posibles riesgos.

Haciendo referencia a las lesiones o días de enfermedad que pudieran disminuir la práctica de actividad físico-deportiva, observamos que la participación de nuestros jóvenes no ha sido afectada por haber recaído durante algún lapso de tiempo. Siendo totalmente opuestos a los comentarios de Sallis et al. (1990) al resaltar que las lesiones limitan la participación de los sujetos. La causa limitante para participar en actividades físico-deportivas a causa de una lesión, resulta más probable en los sujetos que como consecuencia de la participación durante el tiempo, sufre alguna lesión (Carlson et al., 2006; Plotnikoff, 2004; Pollock et al., 1977), siendo los más vulnerables los jóvenes de 18 a 24 años.

Además, los lapsos de lesiones o días de enfermedad en los practicantes no presenta inclinaciones hacia ningún periodo de tiempo en particular, significando que los episodios en los jóvenes son similares, aunque otros autores como Schneider et al. (2006) encuentran que el promedio de inhabilidad son catorce días.

\section{Conclusiones}

Resulta evidente que la promoción de la práctica físico-deportiva debe ser constante, dados los beneficios saludables que promueve a las personas, reduciendo la aparición de enfermedades y dolencias.

Respecto a las hipótesis planteadas constatamos que solamente dos fueron confirmadas. Es decir, en la primera acertamos que las tasas más elevadas corresponden al grupo que práctica actividades físico-deportivas de tiempo libre, seguido por un considerable colectivo que habiendo sido activo ha tenido que abandonar y con bajas tasas en aquellos que nunca han llegado a ser activos. Cifras que presentan bastante similitud a otros estudios en poblaciones universitarias. En relación con la segunda, observamos que la variable sexo es un correlato que se asocia y correlaciona con la práctica, siendo las mujeres quienes menos práctica realizan respecto a los hombres.

En tanto, el resto de las hipótesis no fueron afirmativas, pues comprobamos que las variables edad, Índice de Masa Corporal y días de lesión o enfermedad, no se correlacionan con la práctica de actividad física. Pues estas variables se presentan como contradictorias al ser analizadas como determinantes de la práctica físico-deportiva según los resultados arrojados en diferentes investigaciones.

Esto permitirá fomentar entre las estructuras sociales pertinentes, políticas y universitarias la toma de decisiones para mejorar o adecuar la oferta de programas de práctica de actividades físico-deportivas para estas poblaciones. 
Flores, G.; Ruiz, F.; García, M.E. (2009). Relación de algunos correlatos biológicos y demográficos con la práctica físico-deportiva en estudiantes universitarios. El caso de la Universidad de Guadalajara, México.

\section{Referencias bibliográficas}

Adams-Campell, L.; Washburn, R. y Haile, G. (1990). Physical activity, stress and type a behavior in blacks. Journal of the national medical association, 82, 701.

Barnes, P. y Schoenborn, C. (2003). Informe sobre la actividad física en horas libres y en horas de trabajo en la población estadounidense. U.S. Department of health and Human Services, 333, $1-23$

Benaziza, H. (1998). Creación de asociaciones: una clave para promover la actividad física para la salud. En Informe final. El deporte para todos y los retos educativos a nivel mundial (pp. 186). Barcelona.

Biddle, S. (1993). Psychological benefits of exercise and physical activity. Revista de psicología del deporte, 2, 99-108.

Biddle, S.; Gorey, T. y Stensel, D. (2004). Health-enhancing physical activity and sedentary behaviour in children and adolescents. Journal of sports sciences, 22, 679-701.

Blasco, T., Capdevila, L., Pintanel, M., Valiente, L. y Cruz, J. (1996). Evolución de los patrones de actividad física en estudiantes universitarios. Revista de psicología del deporte, 51-63.

Buendía, L. (1994). El proceso de investigación. En Colás, M. P. y Buendía, L. (Eds.): Investigación educativa. Sevilla: Alfar, 69-107.

Brown, J. D. y Siegel, J. M. (1988). Exercise as a buffer of life stress: a prospective study of adolescent health. Health psychology, 7, 341-353.

Carlson, S.; Hootman, J., Powell, K.; Macera, C.; Heath, G. W.; Gilchrist, J., et al. (2006). Selfreported injury and physical activity levels: United States 2000 to 2002. Annals of epidemiology, 16, 712-719.

Chillón, P.; Tercedor, P.; Delgado, M. y González-Gross, M. (2002). Actividad físico-deportiva en escolares adolescentes. Madrid: Retos. Nuevas perspectivas de educación física, deporte y recreación, 3, 5-12.

Dishman, R. K.; Sallis, J. F. y Orenstein, D. R. (1985). The determinants of physical activity and exercise. Public health reports. 100, 158-171.

Dishman, R. K. y Sallis, J.F. (1994). Determinants and interventions for physical activity and exercise. In C. Bouchard, R.J. Shephard, \& T. Stephens Eds.), Physical activity, fitness, and health: international proceedings and consensus statement (pp. 214-238). Champaign, IL: Human Kinetics.

Dechavanne, N. (1998). El bienestar físico y psíquico en las prácticas físicas. In Informe final. El deporte para todos y los retos educativos a nivel mundial (pp. 121). Barcelona.

Deforche, B.; Bourdeaudhuij, I. y Tanghe, A. (2006). Attitude toward physical activity in normalweight, overweight and obese adolescents. Journal of adolescent health, 38, 560-568.

Estatuto General de la Universidad de Guadalajara. H. Consejo General Universitario, No. 29636, Universidad de Guadalajara, Guadalajara (1994b). Disponible en: http://www. secgral.udg.mx/interface/normatividad/doc_norgral/EstatuotoGen.pdf. [Consulta: 2005, 06 de septiembre].

Figueiredo, M. J. y Rebollo, S. (2003). La actividad física y los hábitos de vida sano: un estudio de la enseñanza técnica del CEFET de Paraíba-Brasil. II Congreso mundial de ciencias de la actividad física y del deporte. (Área 1, Actividad física y salud) (pp. 147-153) [CD ROM]. Granada: Facultad de Ciencias de la Actividad Física y el Deporte.

Granero Gallegos, A.; Ruiz Juan, F. y García Montes, M. E. (2005). El camino de Santiago, una actividad física de recreación y formación para los jóvenes. Madrid: Retos. Nuevas perspectivas de educación física, deporte y recreación, 7, 07-13.

Guillén García, F.; Castro Sánchez, J. J. y Guillén García, M. A. (1997). Calidad de vida, salud y ejercicio: una aproximación al tema desde una perspectiva psicosocial. Revista de psicología del deporte, 91-107. 
Flores, G.; Ruiz, F.; García, M.E. (2009). Relación de algunos correlatos biológicos y demográficos con la práctica físico-deportiva en estudiantes universitarios. El caso de la Universidad de Guadalajara, México.

Revista Internacional de Ciencias del Deporte. 14(5), 59-80. http://www.cafyd.com/REVISTA/01406.pdf

Hernández Rodríguez, A. I. (2001). Análisis de la demanda de la comunidad universitaria almeriense en actividades físico-deportivas. Estudio de adecuación de la oferta. Tesis Doctoral. Universidad de Almería, Almería.

IPAQ. (2002). Cuestionario Internacional de Actividad Física. Versión larga formato auto administrado-últimos 7 días para uso con jóvenes y adultos de mediana edad (15-69 años). Disponible en: http://www.ipaq.ki.se/ [Consulta: 2005, 14 de Junio].

Ho, S. (1987). Assessment of overweight and physical activity among adolescents and youths in Hong Kong. Public health, 101, 457-464.

Instituto Nacional de Estadística Geografía e Informática. (2003). Estadísticas de sector salud y seguridad social. Disponible en: www.inegi.gob.mx [Consulta: 2006, 01 de septiembre].

Jacoby, E., Bull, F. y Neiman, A. (2003). Cambios acelerados del estilo de vida obligan a fomentar la actividad física como prioridad en la Región de las Américas. Revista Panamericana de la Salud, 14, 223-225.

Keating, X.; Guan, J.; Castro, J. y Bridges, D. (2005). A meta-analysis of college students physical activity behaviors. J ournal of American college health. 54, 116-125.

Kelley, G. y Sharpe, K. (1994). Physical activity habits of african-american college students. Research quarterly for exercise and sport, 65, 207-212.

Kilpatrick, M.; Hebert, E. \& Bartholomew, J. (2005). College students' motivation for physical activity: Differentiating men's and women's motives for sport participation and exercise. Journal of American college health, 54, 87-94.

Léon, A.; Connett, J.; Jacobs, D. y Rauramaa, R. (1987). Leisure-time physical activity levels and risk of coronary heart disease and death. The multiple risk factor intervention trial. The J ournal of the American Medical Association. 258, 2388-2395.

Leslie, E.; Owen, N.; Salmon, J.; Bauman, A.; Sallis, J. y Lo, S. K. (1999). Insufficiently active Australian college students: perceived personal, social, and environmental influences. Preventive medicine, 28, 20-27.

Leslie, E., Sparling, P. y Owen, N. (2001). University campus settings and the promotion of physical activity in young adults: lessons from research in Australia and the USA. Health Education, 101, 116-125.

Ley Orgánica de la Universidad de Guadalajara. (1994). Decreto 15319 del Congreso del Estado, publicado en el periódico oficial "El Estado de Jalisco". Disponible en: http://www.secgral.udg.mx/interface /normatividad/doc_norgral/Ley organica.pdf. [Consulta: 2005, 10 de abril].

Organización Mundial de la Salud. (1998). Plan de acción de desarrollo y salud de adolescentes y jóvenes en las Américas, 1998-2001. Disponible en: http://www.who.int/whr/en/whr_es.pdf [Consulta: 2006, 10 de Mayo].

Organización Mundial de la Salud. (2002). Informe sobre la salud en el mundo 2002, promover los riesgos y promover una vida sana. Organización Mundial de la Salud. Disponible en: http://www.who.int/whr/2002/en/whr02_es.pdf [Consulta: 2006, 03 de Julio].

Márquez Ceniceros, E.; Delgado Fernández, D. y López Walle, J. (2003). Hábitos deportivos, de salud, alimenticios, y ocupación del tiempo libre estudiantes de la Universidad Autónoma de Chihuahua, México. En II Congreso Mundial de Ciencias de la Actividad Física y del Deporte. (Área 5, Análisis y Evaluación del Deporte) (pp. 229-233) [CD ROM]. Granada: Facultad de Ciencias de la Actividad Física y el deporte.

Márquez, S.; Rodríguez, J. y Dechavanne, N. (2006). Sedentarismo y salud: efectos beneficiosos de la actividad física. Apunts educación física y deportes, 83, 12-24.

Miller, K.; Staten, R. y Rayens, M. (2005). Levels and characteristics of physical activity among a college student cohort. Research Quarterly for Exercise and Sport, 76, A42.

Parks, Houseman, \& Browson (2003) S.E. Parks, R.A. Houseman and R.C. Browson, Differential correlates of physical activity in urban and rural adults of various socioeconomic backgrounds in the United States. Journal of Epidemiology and Community Health, 57, 29-35. 
Flores, G.; Ruiz, F.; García, M.E. (2009). Relación de algunos correlatos biológicos y demográficos con la práctica físico-deportiva en estudiantes universitarios. El caso de la Universidad de Guadalajara, México.

Pate, R. R.; Pratt, M.; Blair, S. N.; Haskell, W. L.; Macera, C.; Bouchard, C. et al. (1995). Physical activity and public health -- A recommendation from the Centers for Disease Control and Prevention and the American Collage of Sports Medicine. JAMA, 273, 402-407.

Pérez, V. (1998).Criterios de práctica física orientados a la salud. En actas del VII congreso mundial de deporte para todos. El deporte para todos y los retos educativos a nivel mundial (sesión: promoción del bienestar a través del deporte para todos) (pp. 134) [CD ROM]. Barcelona: COI.

Pinto, B. M. y Marcus, B. M. (1995). A stages of change approach to understanding college students physical activity. Journal of American College Health, 44, 27-31.

Plotnikoff, R.; Mayhew, A.; Birkett, N.; Loucaides, C. y Fodor, G. (2004). Age, gender, and urbanrural differences in the correlates of physical activity. Preventive Medicine, 39, 1115-1125.

Pollock, M.; Gettman, L.; Milesis, C.; Bah, M.; Durstine, L. y Johnson, R. (1977). Effects of frequency and duration of training of attrition and incidence on injury. Medicine and science in sports and exercise, 9, 31-6.

Pollock, M. L.; Gaesser, G. A.; Butcher, J. D.; Després, J. P.; Dishman, R. K.; Franklin, B.A. et al. (1998). ACSM Position Stand: The recommended quantity and quality of exercise for developing and maintaining cardiorespiratory and muscular fitness, and flexibility in healthy adults. Medicine and science in sports and exercise, 30, 975-991.

Rodríguez, M.; Castañeda, A.; Correa, L. y Sarabia, M. (2004). Estilos de vida saludables de los estudiantes de la Corporación Universitaria Lasallista. Revista Lasallista de Investigación, 1, 3641.

Ruiz Juan, F.; García Montes, M. E. y Hernández A. I. (2001). El interés por la práctica de actividades físico-deportivas de tiempo libre del alumnado de la Universidad de Almería. Un estudio longitudinal. Apunts. Educación Física y Deportes, 63, 85-92.

Ruiz Juan, F. y García Montes, M. E. (2005). Modalidades de práctica de actividades físicodeportivas de tiempo libre. Estudio comparativo entre el alumnado de Enseñanza Secundaria Post-obligatoria y de segundo ciclo de la Universidad de Almería. Tandem. Didáctica de la Educación Física, 18, 79-94.

Ruiz Juan, F.; García Montes, M. E. y Gómez López, M. (2005). Hábitos físico-deportivos en centros escolares y universitarios. Madrid: Gymnos.

Sallis J. y Owen, N. (1999). Physical activity and behavioral medicine. Thousand Oaks, CA: Sage Publications, 1999, pp. 110-134.

Sallis, J.; Hovell, M.; Hofstetter, R.; Elder, J.; Faucher, P.; Spry, V., et al. (1990). Lifetime history of relapse from exercise. Addictive Behaviors, 15, 573-579.

Sallis, J.; Hovell, M. y Hofstetter, R. (1992). Predictors of adoption and maintenance of vigorous physical activity in men and women. Preventive Medicine, 21, 237-251.

Sallis, J. (1999). Influences on physical activity of children, adolescents, and adults. President's Council on Physical Fitness and Sport. Research Digest, 1 [7].

Sallis, J. (2000). Age-related decline in physical activity: A synthesis of human and animal studies. Medicine and science in sports and exercise, 32, 1598-1600.

Sánchez Pérez, A.; García Domínguez, F.; Landabaso, V. y Nicolás Martínez, L. D. (1998). Participación en actividad física de una muestra universitaria a partir del modelo de las etapas de cambio en el ejercicio físico: un estudio piloto. Revista de Psicología del Deporte, 7, $233-245$.

Shephard, R. J. y Cox, M. (1980). Some characteristics of participants in an industrial fitness programme. Canadian Journal of applied sport sciences. 2, 69-76.

Schmitz, K.; French, S. y Jeffery, R. (1997). Correlates of changes in leisure time physical activity over 2 years: The healthy worker project. Preventive Medicine, 26, 570-579.

Schneider, S.; Seither, B.; Tonges, S. y Zimmermann-Stenzel, M. (2006). Sport injuries: population based representative data on incidence, diagnosis, sequelae, and high risk groups. British Journal of Sport Medicine, 54, 233. 
Flores, G.; Ruiz, F.; García, M.E. (2009). Relación de algunos correlatos biológicos y demográficos con la práctica físico-deportiva en estudiantes universitarios. El caso de la Universidad de Guadalajara, México.

Schmitz, K.; Lytle, L.; Phillips, G.; Murray, D.; Birnbaum, A. y Kubik, M. (2002). Psychosocial correlates of physical activity and sedentary leisure habits in young adolescents: The teens eating for energy and nutrition at school study. Preventive Medicine, 34, 266-278.

Telama, R. y Yang, X. (2000). Decline pf physical activity from youth to young adulthood in Finland. Medicine and science in sports and exercise, 32, 1617-1622.

Trost, S.G.; Owen, N.; Barman, A.E.; Sallis, J.F. y Brown, W. (2002). Correlates of adults' participation in physical activity: review and update. Medicine and science in sports and exercise, 34, 12, 1996-2001.

U.S Department of Health and Human Services. (1996). Physical activity and Health: a report of the surgeon general. U.S Department of Health and Human Services. Disponible en http://www.cdc.gov. [Consulta: 2006, 22 de junio].

U.S Department of Health and Human Services. (2006). Body mass index: About BMI for adults. U.S Department of Health and Human Services. Disponible en: http://www.cdc.gov/nccdphp/dnpa/bmi/adult_BMI/about_adult_BMI.htm [Consulta: 2006, 10 de Julio].

Universidad de Guadalajara. (2004). Alumnos inscritos a Centros Universitarios en el 2004b.

Varo Cenarruzabeitia, J. J.; Martínez-González, M. A. y Martínez Hernández, J. A. (2003). Beneficios de la actividad física y riesgos del sedentarismo. Medicina Clínica, 121, 665-672.

Von Bothmer, M. y Fridlun, B. (2005). Gender differences in health habits and in motivation for a healthy among Swedish university students. Nursing and Health Sciences, 7, 107-118. 